\title{
Characteristics Features of Hepatocellular Carcinoma in Bangladesh and their Public Health Implications
}

\author{
ABM Shakil Gani, Mamun AI-Mahtab, Salimur Rahman, Sheikh Mohammad Fazle Akbar
}

\begin{abstract}
Background/Objectives: In spite of being a common cancer, little is known about clinical presentation, etiological factors and prognosis of hepatocellular carcinoma (HCC) in Bangladesh. Accordingly, early detection and efficient management of HCC is not available at this country.
\end{abstract}

Materials and methods: Demographical data, etiological factors and clinical presentations of 57 consecutive patients with $\mathrm{HCC}$ were analyzed prospectively at the only medical university of Bangladesh.

Results: Ninety-one percent patients with HCC were male. The mean age of HCC patients was 45.8 years (standard deviation: 15.3 years; range: $18-75$ years). All patients were symptomatic when they first appeared to the physicians, and many of them were unaware that they had been suffering from liver diseases. Hepatitis B surface antigen was detected in $62 \%$ and antibody to hepatitis $\mathrm{C}$ virus was found in $6 \%$ patients with $\mathrm{HCC}$. Most of the patients with HCC had liver cirrhosis (77\%). Single HCC nodule was detected in $61 \%$ patients. Sixty-four percent patients had intermediate stage of liver cancer.

Conclusion: Containment of HCC-related morbidity and mortality and better prognosis of HCC in Bangladesh depends on early diagnosis of hepatitis viruses.

Keywords: Hepatocellular carcinoma, Hepatitis B virus, Hepatitis C virus, Advanced hepatocellular carcinoma.

How to cite this article: Gani ABMS, Al-Mahtab M, Rahman S, Akbar SMF. Characteristics Features of Hepatocellular Carcinoma in Bangladesh and their Public Health Implications. Euroasian J Hepato-G astroenterol 2013;3(1):28-30.

\section{Source of support: Nil}

Conflict of interest: None

\section{INTRODUCTION}

Primary liver cancer, which consists predominantly of hepatocellular carcinoma ( $\mathrm{HCC}$ ), is the fifth most common cancer worldwide and the third most common cause of cancer mortality. ${ }^{1}$ Globally, over half a million people develop HCC each year and an almost equal number die of it. $^{2}$ HCC has several characteristics epidemiologic features including dynamic temporal trends; marked variations among geographic regions, racial and ethnic groups, and between men and women; and the presence of several welldocumented potentially preventable risk factors. Studies have shown that HCC largely occurs within an established background of chronic liver disease and cirrhosis. Major causes of cirrhosis in patients with HCC include hepatitis $B$ virus (HBV), hepatitis C virus (HCV), alcoholic liver disease and possibly nonalcoholic steatohepatitis (reviewed in details in reference 3 ). In addition, hereditary hemochromatosis, alpha-antitrypsin deficiency, autoimmune hepatitis, and some porphyrias have also been associated as minor etiological factors in some patients with $\mathrm{HCC}^{3}$ Improved insights about pathogenesis, clinical features, prognosis and interventional strategies have led to develop mechanisms of early diagnosis and improved management against HCC in most developed countries. ${ }^{4} \mathrm{H}$ owever, this success has not been duplicated worldwide. ${ }^{5,6}$ A lthough most patients with $\mathrm{HCC}$ reside in developing countries of the world, there is lack of information about etiological factors, clinical presentation and management of these patients in the developing countries.

$B$ angladesh is a developing country in A sia with a population of 140 million. It is estimated that more than 8 million people are chronically infected with $\mathrm{HBV}^{7}$ and about 1 to 2 million with $\mathrm{HCV} .{ }^{8} \mathrm{H}$ ow ever, there are paucity of information about clinical and pathological features of $\mathrm{HCC}$ in $\mathrm{B}$ angladesh. $\mathrm{K}$ han et al have detected liver cirrhosis in $18.5 \%$ patients with HCC by examining a cohort of 64 patients at $\mathrm{B}$ angladesh in $1997 .{ }^{9} \mathrm{~A}$ nother study conducted in United K ingdom, among HCC patients of Bangladeshi origin, has shown that 36 and $56 \%$ of these patients have been infected with HBV and HCV, respectively. ${ }^{10} \mathrm{H}$ owever, studies from India, a close neighbor of $B$ angladesh, have reported that about $80 \%$ HCC patients are infected with HBV and most of the HCC patients are cirrhotic. ${ }^{11}$ These studies indicate that more investigations are required to develop insights about clinical features, etiological agents and epidemiology of HCC at B angladesh.

The present communication, a prospective study, was accomplished to understand the present status of HCC in $B$ angladesh. We took elaborative history of HCC patients, and also checked clinical profiles and etiological agents of HCC patients. The findings of this study would be discussed for devel opment a strategy for containment of incidence of HCC and also for better management of these patients in Bangladesh.

\section{PATIENTS AND METHODS}

\section{Patients}

Consecutive 57 patients with $\mathrm{HCC}$ those attended the Bangabandhu Sheikh M ujib M edical University, Dhaka, 
Bangladesh, in 2008 were enrolled in this study. This is the only Postgraduate Medical University of Bangladesh and acts as a referral center for patients with intractable diseases. The mean age of the patient was 45.8 years (standard deviation: 15.3 years). Fifty-two patients were male (91\%) and the rest five were female $(9 \%)$.

\section{Study Design}

A questionnaire was prepared that had two parts: (1) B aseline characteristics of the patients with HCC, and (2) presenting symptoms of the patients. This was filled up by a clinical monitor by asking questions to the patients. The patients were examined by the attending physician. Presence of hepatitis B surface antigen ( $\mathrm{HBsA}$ ) and anti-HCV antibody was evaluated using commercial kits. The diagnosis of underlying cirrhosis was done on the basis of clinical, endoscopic findings (presence of varices) and radiological features (ultrasonography and computed tomography). The presence of HCC was confirmed by either ultrasound (US), or computed tomography, or magnetic resonance imaging of the abdomen. In some cases, more than one imaging technique was employed. The B arcelona Clinic L iver Cancer (BCLC) staging of HCC were adopted on the basis of clinical and radiological information. ${ }^{12}$ The confirmation of HCC was done by cytopathological examination of fine needle aspirates. Patients were excluded from this study if cytopathological study could not provide a confirmatory diagnosis of HCC. The study was conducted according to the ethical guidelines of $B$ angabandhu Sheikh $M$ ujib M edical U niversity, D haka, B angladesh, and is in agreement with those set out by the 1975 Declaration of Helsinki as revised in 1983.

\section{RESULTS}

The baseline characteristics of patients with HCC are presented in Table 1. The mean age of HCC patients was 45.81 years, and most of them were male ( 52 of 57 patients, $91 \%$ ). A bout $40 \%$ of the patients were farmer by profession. It was also found that more than $60 \%$ were attending the hospital of Dhaka from rural areas. Some of the patients travelled more than $300 \mathrm{~km}$ to get treatment.

The presenting symptoms of the patients have been shown in Table 2. The patients experienced various symptoms for different duration, but, this was their first consultation with a recognized medical doctor. The symptoms that they experienced before attending this hospital include loss of body weight, pain in the abdomen, ascites and jaundice. Accordingly, all patients with HCC had some symptoms during their first presentation. Some

of the patients had multiple symptoms. M ost of the patients had abdominal pain or anorexia as presenting symptoms. A bout $49 \%$ patients felt a palpable mass at the abdomen. J aundice was detected in $15 \%$ patients. When the patients were checked by attending physicians, a palpable liver was found in $74 \%$ patients. A scites were detected in $57 \%$ patients. Edema was seen in 23\% patients (Table 3).

Imaging technique reveal ed space-occupying lesions in, all but two patients with HCC (96\%, 53 of 55 patients). The numbers of lesions were single in $61 \%$ patients and multiple in $39 \%$ patients. Evidence of liver cirrhosis was seen in $77 \%$ patients by ultrasonography and assessment of liver function test. M ost of patients with liver cirrhosis were

\begin{tabular}{|c|c|}
\hline Total patients & 57 patients \\
\hline \multicolumn{2}{|l|}{ Age } \\
\hline $\begin{array}{l}\text { Mean } \pm S D \\
\text { Range }\end{array}$ & $\begin{array}{l}45.81 \pm 15.31 \text { years } \\
18-75 \text { years }\end{array}$ \\
\hline \multicolumn{2}{|l|}{ Sex } \\
\hline $\begin{array}{l}\text { Male } \\
\text { Female }\end{array}$ & $\begin{array}{l}9 \% \\
9 \%\end{array}$ \\
\hline \multicolumn{2}{|l|}{ Occupation } \\
\hline Farming & $41 \%$ \\
\hline Business & $17 \%$ \\
\hline Housewife & $10 \%$ \\
\hline Service & $9 \%$ \\
\hline Student & $9 \%$ \\
\hline Others & $14 \%$ \\
\hline
\end{tabular}

Note: Age, sex and occupation of patients with HCC are shown

Table 2: Presenting symptoms of patients with HCC

\begin{tabular}{ll} 
Presenting symptoms & Percentage \\
\hline Mass in abdomen & 49 \\
Pain & 79 \\
Weight loss & 45 \\
Fever & 53 \\
Anorexia & 79 \\
Jaundice & 15 \\
Cachexia & 11 \\
Anemia & 32 \\
\hline
\end{tabular}

Note: Some of the patients with HCC had multiple symptoms at first presentation

Table 3: Clinical features of patients with HCC

$\begin{array}{ll}\text { Leukonychia } & 11 \% \\ \text { Edema } & 23 \% \\ \text { Gynecomastia } & 13 \% \\ \text { Spider telangiectasia } & 32 \% \\ \text { Palmar erythema } & 11 \% \\ \text { Palpable liver } & 74 \% \\ \text { Palpable spleen } & 23 \% \\ \text { Ascites } & 57 \% \\ \text { Testicular atrophy } & 46 \% \\ \text { Features of metastasis (lungs) } & 2 \%\end{array}$


grouped into Child-Pugh score B (43\%). Sixty-four percent patients had intermediate stage of $\mathrm{HCC}$, according to the grading of the grading of BCLC.

Regarding etiological factor, $62 \%$ patients were expressing HBsA g in the sera, whereas, anti-HCV antibody was detected in $6 \%$ patients. N one of the patients has any history of excessive alcohol consumption.

\section{DISCUSSION}

The study presented here has shown that most of the patients with HCC at Bangladesh were infected with the HBV and about $77 \%$ of these patients were suffering from liver cirrhosis. Although the results of the present study are not in line with previous publications in 1990s about HCC in $B$ angladesh, ${ }^{9,10}$ the data presented here are in line with that what has been reported by K umar et al about etiology and cirrhosis of HCC patients in India, ${ }^{11}$ a close neighbor of Bangladesh.

In addition, this study revealed that proper screening of liver cirrhosis is absent in Bangladesh. M ost of the patients were unaware of their chronic liver diseases before attending the tertiary hospitals. A II patients presented with complain like abdominal pain, weight loss, fever, jaundice and pal pable mass in the abdomen. The extent of liver cirrhosis was also in progressive state. Unfortunately, most of the patients attended the physicians after development of HCC.

These facts unveil the importance of diagnosis and follow-up of patients with chronic viral liver diseases at an early stage. A s most of these patients had advanced HCC, this will comprise their ultimate survival. However, single HCC nodule was detected in $61 \%$ patient. If proper interventional strategies can be adopted in these patients, better survival can be expected in these patients.

\section{CONCLUSION}

HCC in Bangladesh occurs at a comparatively earlier age and most of them reside in rural areas. N one of these patients had any idea of their liver diseases prior to diagnosis of HCC at tertiary level hospital. When symptoms were developed due to advanced HCC, they came to consult with physicians. As most of the patients had liver cirrhosis, a mechanism should be developed at $B$ angladesh to properly diagnose chronic hepatitis virus infection and liver cirrhosis. This would lead to better survival of HCC patients.

\section{REFERENCES}

1. El-Serag HB, Rudolph KL. Hepatocellular carcinoma: Epidemiology and molecular carcinogenesis. Gastroenterology 2007;132:2557-76.
2. Perz JF, A rmstrong GL, Farrington $L A, H$ utin $Y J, B$ ell $B P$. The contributions of hepatitis $B$ virus and hepatitis $C$ virus infections to cirrhosis and primary liver cancer worldwide.J Hepatol 2006; 45:529-38.

3. Hussain K, El-Serag HB. E pidemiology, screening, diagnosis and treatment of hepatocellular carcinoma. M inerva Gastroenterol Dietol 2009;55:123-38.

4. Kudo M. The 2008 Okuda lecture: M anagement of hepatocellular carcinoma: From surveillance to molecular targeted therapy. J Gastroenterol Hepatol 2010;25:439-52.

5. O mata M, Dan Y, Daniele B, Plentz R, Rudolph K L, M anns M, et al. Clinical features, etiology and survival of hepatocellular carcinoma among different countries. J Gastroenterol Hepatol 2002;17 Suppl:S40-49.

6. Ogunbiyi J 0 . Hepatocellular carcinoma in the developing world. Semin Oncol 2001;28:179-87.

7. Mahtab MA, Rahman S, Karim MF, K han M, Foster G, Solaiman $S$, et al. Epidemiology of hepatitis $B$ virus in Bangladeshi general population. Hepatobilliary Pancreat Dis 2008; 7:595-600.

8. K han $M, Y$ ano $M$, Hashizume $K$, Y ousuf $M$, Tanaka $E$, et al Comparison of seroepidemiology of hepatitis $C$ in blood donors between Bangladesh and Japan. Gastroenterol J pn 1993;28: 28-31.

9. K han M, Haq SA, A hmed N, M atin M A. Etiology and clinical profile of hepatocellular carcinoma in Bangladesh. B angladesh Med Res Counc Bull 1997;23:16-24.

10. Zaman S, K han M, A lam K, W illiams R. Primary hepatocellular carcinoma and viral hepatitis $B$ and $C$ infection in Bangladeshi subjects. J Trop M ed Hyg 1995;98:64-68.

11. Kumar R, Kumar SM, Chander BS, Sakhuja P, Sarin SK. Characteristics of hepatocellular carcinoma in India: A retrospective analysis of 191 cases. QJ M 2008;101:479-85.

12. Bruix J, Sherman $M$, Llovet J $M$, Beaugrand $M$, Lencioni $R$, Burroughs AK, et al. Clinical management of hepatocellular carcinoma. Conclusions of the Barcelona-2000 EA SL conference. European A ssociation for the Study of the Liver. J Hepatol 2001;35:421-30.

\section{ABOUT THE AUTHORS}

\section{ABM Shakil Gani}

Department of Hepatology, Bangabandhu Sheikh M ujib M edical University, Shahbag, D haka, B angladesh

\section{Mamun Al-Mahtab (Corresponding Author)}

Department of Hepatology, Bangabandhu Sheikh Mujib Medical University, Shahbag, Dhaka-1000, Bangladesh, e-mail: shwapnil@agni.com

\section{Salimur Rahman}

Department of Hepatology, Bangabandhu Sheikh M ujib M edical University, Shahbag, D haka, B angladesh

\section{Sheikh Mohammad Fazle Akbar}

Department of M edical Sciences, Toshiba General Hospital, Higashi Tokyo, Japan 